\title{
Reversible cerebral shrinkage in kwashiorkor: an MRI study
}

\author{
Geney D Gunston, Dawn Burkimsher, Hester Malan, Alan A Sive
}

\begin{abstract}
Protein energy malnutrition is associated with cerebral atrophy which may be detrimental to intellectual development. The aim of this study was to document the anatomical abnormalities which lead to the appearance of cerebral atrophy using magnetic resonance imaging (MRI) in the acute stage of kwashiorkor and to monitor changes during nutritional rehabilitation.

Twelve children aged 6 to 37 months requiring admission to hospital for the treatment of kwashiorkor were studied. The children were evaluated clinically, biochemically, and by MRI of their brains on admission and 30 and 90 days later. Brain shrinkage was present in every child on admission. White and grey matter appeared equally affected and the myelination was normal for age. At 90 days, the cerebral changes had resolved in nine and improved substantially in the remainder, by which time serum proteins and weight for age were within the normal range. The findings of this study suggest that brain shrinkage associated with kwashiorkor reverses rapidly with nutritional rehabilitation.
\end{abstract}

(Arch Dis Child 1992;67:1030-1032)

Protein energy malnutrition is an important nutritional deficiency world wide. In the last $\mathbf{4 0}$ years there has been significant progress in treating the acute effects of nutritional diseases, but the consequence of severe protein energy malnutrition on the developing brain is still ill defined. ${ }^{1}$ A World Health Organisation (WHO) symposium concluded that despite the widely held opinion that malnutrition in early life jeopardises long term mental development, the evidence to support this opinion, especially evidence in man, is scanty. ${ }^{2}$

Stoch and Smythe followed up marasmic children for 15 years from infancy to investigate the effect of food deprivation on intellectual development. ${ }^{34}$ They concluded that intellectual performance was impaired in the undernourished group when compared with controls. As the control children came from relatively stable home conditions, environmental factors may have accounted for the difference in outcome between the groups. Winick has stressed that undernutrition does not occur in isolation, but is part of a complex mixture of risk factors of genetic and environmental origin affecting subsequent development. ${ }^{5}$ To overcome the environmental differences, Bowie et al used normally nourished siblings of the affected children as controls. ${ }^{6}$ These siblings had grown up under the same environmental circumstances as the study subjects. In a prospective 15 year follow up of children with kwashiorkor no differences in educational achievement were noted between the study subjects and their siblings.

The affective changes which are a hallmark of the acute phase of kwashiorkor are accompanied by structural changes in the brain. Househam and de Villiers reported cerebral atrophy in 12 children using computed tomography. ${ }^{7}$ Subsequently Househam found that the atrophy had resolved on scans performed an average of 14 months later. ${ }^{8}$ He concluded that atrophy was apparent and suggested that "cerebral shrinkage' more aptly describes the abnormalities.

The aim of this study was to document and define the anatomical abnormalities in the brains of children with kwashiorkor using magnetic resonance imaging (MRI). As MRI is safe and non-invasive, repeat scans could be performed 30 and 90 days later to monitor the sequence of changes during nutritional rehabilitation.

Patients and methods

Twelve children admitted to hospital for the treatment of kwashiorkor were studied; there were nine boys and three girls with a median age of 14.5 months (range 6-37 months). The diagnosis conformed to the criteria of the Wellcome classification of infantile malnutrition. ${ }^{9}$ Children with a history of convulsions or previous disease involving the central nervous system were excluded.

We assessed the children to have mild, moderate, or severe disease on the basis of their clinical findings. Thus, children with mild disease had hair changes (straightening and lightening), dry skins, minimal pitting oedema, hepatomegaly of less than $3 \mathrm{~cm}$ below the costal margin, and no superadded infections. Children with moderate disease had dry generalised skin changes and nappy rash, hair changes, moderate pitting oedema, hepatomegaly greater than $3 \mathrm{~cm}$ below the costal margin, and superadded infections. Children with severe disease had raw, weeping skin lesions, hair changes, marked pitting oedema with swollen palms and soles, hepatomegaly greater than $3 \mathrm{~cm}$ below the costal margin, and superadded infections (for example, diarrhoeal disease, pneumonia, otitis media).

On admission to hospital, the weight, height, and head circumference of each child was documented and a blood sample was taken for 
estimation of serum proteins and any other investigations necessary for the clinical care of the child. MRI of the brain was carried out within five days of admission. The clinical and biochemical measurements and MRI scans were repeated 30 and 90 days later.

Table 1 Weight, head circumference, and serum proteins on admission and during recovery. Figures are mean $(S D)$

\begin{tabular}{|c|c|c|c|}
\hline & Admission & Day 30 & Dav 90 \\
\hline $\begin{array}{l}\text { Weight }(\mathrm{kg}) \\
\text { Expected weight }(\%) \\
\text { Head circumference }(\mathrm{cm}) \\
\text { Total protein }(\mathrm{g} / \mathrm{l}) \\
\text { Albumin }(\mathrm{g} / \mathrm{l})\end{array}$ & $\begin{array}{l}8 \cdot 44(1 \cdot 82) \\
75 \cdot 6(9 \cdot 22) \\
45 \cdot 9(1 \cdot 89) \\
41 \cdot 8(4 \cdot 77) \\
17 \cdot 8(3 \cdot 4)\end{array}$ & $\begin{array}{l}9 \cdot 35(2 \cdot 08) \\
81 \cdot 4(9 \cdot 55) \\
46 \cdot 4(2 \cdot 25) \\
66 \cdot 8(9 \cdot 83) \\
35 \cdot 2(6 \cdot 9)\end{array}$ & $\begin{array}{l}10 \cdot 40(1 \cdot 82) \\
95 \cdot 4(8 \cdot 78) \\
46 \cdot 7(1 \cdot 80) \\
67 \cdot 4(6 \cdot 33) \\
37 \cdot 2(3 \cdot 3)\end{array}$ \\
\hline
\end{tabular}
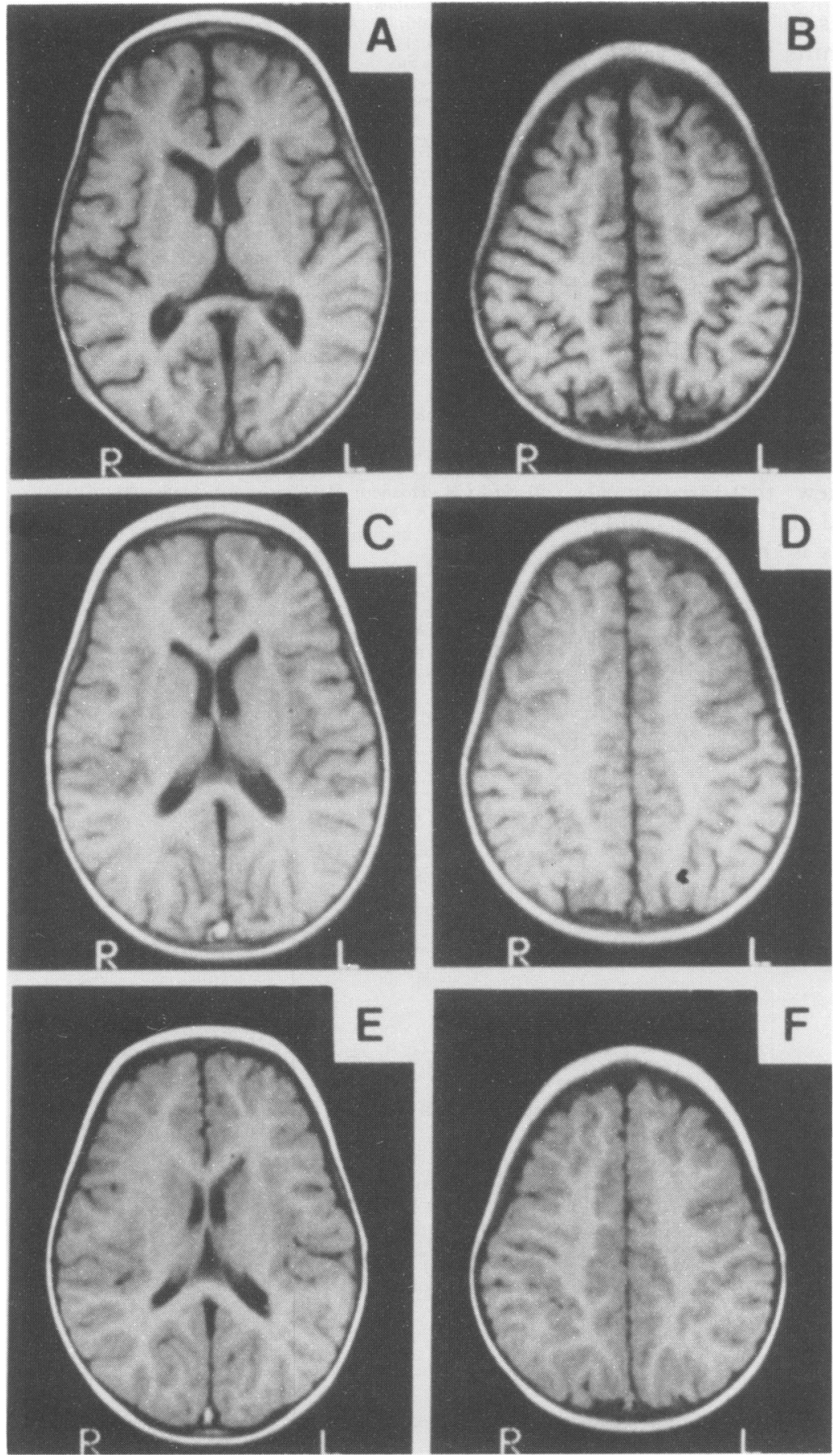

$A$ and $B$ illustrate the typical MRI changes of cerebral atrophy as seen on admission. $C$ and $D$ show the improvement by day 30.E and $F$ are day 90 scans, illustrating the rapid resolution of the signs of cerebral atrophy with refeeding (patient 4$) . A, C$, and $E$ are mid and $B, D$, and $F$ are high axial views of the brain.
Table 2 Distribution of patients according to the degree of cerebral atrophy as assessed by MRI over time

\begin{tabular}{llll}
\hline Extent of cerebral atrophy & \multicolumn{3}{l}{ No of patients } \\
\cline { 2 - 4 } & Admission & Day 30 & Day 9. \\
\hline Moderate & 5 & 2 & 0 \\
Mild to moderate & 6 & 2 & 0 \\
Mild & 1 & 8 & 3
\end{tabular}

Infants were fed a commercial soya formula and a lactose free diet for the first 10 days. Additional folate, multivitamins, and trace metals were given. Iron was withheld during this period. Thereafter a mixed Western style diet containing cows' milk was gradually introduced. Patients received antibiotics according to laboratory and clinical indications. After discharge from hospital, supervised nutritional rehabilitation was continued in a convalescent facility.

Consent for the study was obtained from the escorting parent of each child before scans were performed. The study was approved by the ethics and research committee of the Medical Faculty of the University of Cape Town. No child requiring an MRI scan for a valid clinical indication was denied access by a child with kwashiorkor in whom the MRI scan was considered a research procedure.

\section{Results}

Of the 12 children studied, nine had moderate and three had severe disease. Head circumference was measured on admission in 11 , and was within the normal range for 10 children. Six children had diarrhoea, one had pneumonia, and four had both. None of the children had meningitis or an infection of the central nervous system. There was an increase in weight and serum proteins over the 90 day period to within the normal range for age (table 1 ).

All the children had abnormal MRI scans of the brain. The abnomalities included widened cortical sulci (frontal, high parietal, sylvian fissures); widened interhemispheric fissure and cerebellar folia; enlarged ventricles (especially the frontal horns of lateral ventricles), and enlarged basal cisterns. The changes were compatible with 'cerebral atrophy' and were designated mild, mild to moderate, or moderate. In one child the changes were mild, in six they were mild to moderate, and in five they were moderate. By day 30, the majority of children showed an improvement in the degree of cerebral atrophy and by day 90 the changes had resolved in the majority (table 2 ). The figure shows the sequence of changes in a representative patient. Grey and white matter were equally affected and myelination appeared to be appropriate for age. ${ }^{10}$

There was no clear relationship between the clinical severity of the kwashiorkor or the serum albumin concentration on admission and the degree of cerebral shrinkage.

\section{Discussion}

MRI clearly demonstrated cerebral shrinkage in the acute stage of kwashiorkor in our patients. 
Brain scans improved with refeeding and complete resolution occurred by 90 days in nine of the 12 patients. The underlying reason for the cerebral shrinkage is unclear and grey and white matter appeared equally affected.

In rats subjected to severe protein restriction, the lipid content of the brain has been shown to decrease. ${ }^{11}$ Therefore, it may be postulated that loss of myelin lipid accounts for the cerebral shrinkage seen in our patients and it is feasible that restoration of lipid to the myelin membrane with refeeding accounts for the reversal of the cerebral shrinkage. However, in our study, the myelination was assessed as appropriate for age.

Improvement in brain size was apparent on day 30 of nutritional rehabilitation in the majority of patients. This rapid improvement suggests that the changes in size may relate predominantly to fluid shifts between various compartments and to a lesser degree to fat or protein abnormalities in the brain. Heinz et al have reported 'reversible cerebral atrophy' in patients with anorexia nervosa and propose that fluid shifts account for the phenomenon. ${ }^{12}$ Fluid moves out of intravascular spaces as a result of decreased colloid osmotic pressure and floods the subarachnoid spaces, dilates the ventricles, and widens the cisternal spaces and sulci. When nutrition is improved the plasma proteins rise, the extracellular fluid moves back into the intravascular space.

Taken together with studies of intellectual function using carefully selected controls, our findings support the view that anatomical recovery is relatively rapid and that the child's ultimate intellectual status is the result of the interaction between a complex mixture of risk factors of genetic and environmental origin. If environmental factors and social stimulation at the time of undernutrition are favourable, the chances of retarded development and long term adverse effects are diminished.

As the environment plays a crucial part in determining the outcome of the developmental process, the rehabilitation of children with kwashiorkor must include in addition to adequate nutrition, stimulation to provide for intellectual and emotional growth.

This investigation was supported by the Bernard and Rita Brodie Associateship in Nutrition and the Research Insitute for Medical Biophysics of the South African Medical Research Council. We Biophysics of the South African Medical Research Council. We
wisk to thank the staff of the paediatric wards for the day to day wisk to thank the steff
care of the children.

1 Hansen JDL. Malnutrition review. Pediatric Reviews and Communications 1990;4:201-12

2 World Health Organisation. WHO symposium: malnutrition and mental development. WHO Chronicle 1974;28:95-102.

3 Stoch MB, Smythe PM. The effect of undernutrition durin infancy on subsequent brain growth and intellectual development. S AfrMed f 1967;41:1027-30.

4 Stoch MB, Smythe PM. 15-year development study on effects of severe undernutrition during infancy on subsequen

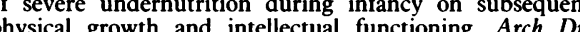
Child 1976;51:327-36.

5 Winick $M$. The role of early nutrition in subsequent development and optimal future health. Bull NY Acad Med 1989; 65:1020-5.

6 Bowie MD, Moodie AD, Mann MD, Hansen JDL. A prospective 15-year follow-up study of kwashiorkor patients. Part I: physical growth and development. $S$ Afr Med $\mathcal{J}$ Part I: physical

7 Househam KC, de Villiers JFK. Computed tomography in severe protein energy malnutrition. Arch Dis Child 1987;62: 589-92.

8 Househam KC. Computed tomograpy of the brain in kwashiorkor: a follow up study. Arch Dis Child 1991;66: 623-6.

9 Anonymous. Classification of infantile malnutrition [Editorial]. Lancet 1970;ii:302-3.

10 Bird CR, Hedberg M, Drayer BP, Keller PJ, Flom RA, Hodak JA. MR assessment of myelination in infants and children: usefulness of marker sites. AfNR 1989;10: children:

11 Benton JW, Moser HW, Dodge PR, Carr S. Modification of myelination in the rat by early nutritional deprivation. myelination in the rat by
Pediatrics 1966;38:801-7.

12 Hein $\%$ ER, Martinez J, Haenggeli A. Reversibility of cerebral atrophy in anorexia nervosa and Cushing's syndrome. f Comput Assist Tomogr 1977;1:415-8. 